\title{
Acute paraplegia due to an extradural spinal lipoma: Case report
}

\author{
YV Meisheri, S Mehta and K Chattopadhyay \\ Department of Medicine, GS Medical College and King Edward Memorial Hospital, Parel, Bombay 400012, \\ Maharashtra, India
}

\begin{abstract}
We describe a 20 year old man, who presented with an acute onset of transverse myelopathy evolving over $24 \mathrm{~h}$ at T6 spinal level; as yet, an unreported presenting symptom from a midthoracic intraspinal lipoma. The C.S.F findings suggested a spinal block. MRI, imaging was not practical. Urgent myelography revealed extradural compression at the T5-6 vertebral level. The patient underwent surgical debulking of the tumor which on histopathology was found to be a lipoma, but there was no neurological improvement even at 3 months follow up. We believe that patients with intraspinal lipomas are at high risk of developing irreversible neurological dysfunction.
\end{abstract}

Keywords: acute transverse myelopathy; spinal neoplasm; thoracic lipoma

\section{Case report}

A 20 year old man was admitted to our hospital with the acute onset of motor weakness of both lower limbs, total sensory loss below T6 spinal level, and incontinence of urine and faeces. Four days prior to admission he experienced tingling in both legs, and a sense of heaviness the same evening. The next morning he was unable to move his lower limbs which were completely numb, and had dribbling of urine and incontinence of faeces. There was no antecedent history of root pains, vertebral pain, transient or prolonged limb disability, fever or trauma. No history of excessive steroid therapy, injections of vaccines or sera or of surgery for meningocoele in childhood. General examination revealed an afebrile, normotensive patient with no congenital cranial, spinal, appendiceal, skeletal or soft-tissue anomaly. Neurological examination showed a flaccid paraplegia with loss of plantar, ankle, knee, cremasteric and abdominal reflexes, and total loss of all modalities of sensation below T6 dermatome. He had a distended, palpable bladder with overflow incontinence. The upper limbs and the cranial nerves were normal. Examination of heart, lungs and abdomen was unremarkable.

A clinical diagnosis of acute non-compressive transverse myelopathy of (1) viral (2) demyelination cause was entertained. Routine biochemical and hematological investigations were normal. Serum VDRL, HIV and HBsAg tests were negative. Roentgenograms of the spine were normal except for minimal mid-thoracic rightward scoliosis involving T57 vertebrae. On lumbar puncture there was xanthochromic C.S.F., with an opening and closing pressure

Correspondence: YV Meisheri of $120 \mathrm{~mm}$ and $100 \mathrm{~mm}$ respectively. C.S.F. analysis showed a protein $243 \mathrm{mg} / \mathrm{dL}$, cells 8 lymphocytes/ $\mathrm{cmm}$, sugar $60 \mathrm{mg} / \mathrm{dL}$, chlorides $660 \mathrm{mg} / \mathrm{dL}$ and negative VDRL. Thus there was a spinal block and acute compressive myelopathy. Urgent myelography was done on day 2, through a lateral cervical puncture, revealing almost total spinal block T5-6 vertebral level, with deviation of right edge medially, suggesting extradural compression.

\section{Operation}

The patient underwent a mid-thoracic laminectomy from T5-7 and partial T8 laminectomy. An extensive thick layer of avascular, partially encapsulated, nonlobulated fibrofatty tissue with ill-defined margins was found extending from T4-9 levels. It was nonadherent and did not compress the dura throughout the entire tumour extent. The mass could be easily dissected. There was no meningeal defect, nor a stalk deep to the tumor, nor any intramedullary extension of the fibrofatty tissue. After excision, the dura was found to be intact and pulsatile.

\section{Pathological report}

The specimen consisted of several portions, aggregating $2 \mathrm{~cm} \times 1 \mathrm{~cm}$ of firm, grayish brown tissue. Microscopic study showed well differentiated adipose tissue and fibrous strands, suggestive of lipoma.

\section{Post-operative course}

There was no neurological improvement even after early surgery. He remains totally paraplegic even at 3 months follow up. 


\section{Discussion}

Spinal cord lipomas are uncommon, accounting for approximately $1 \%$ of all spinal cord tumours. ${ }^{1}-5 \mathrm{~A}$ cervicodorsal lipoma is usually reported to be intradural, with no gender predilection. The mean age of onset at 36 years, urinary dysfunction occurs in $25 \%$. A subcutaneous lipoma at the vertebral site is seen in $10 \%$ of patients. Typically there is a slow progressive history over years or months with spastic paraplegia. ${ }^{4.8}$ Unlike reported cases of extradural spinal lipomas, our patient was a 20 year old otherwise healthy man with no antecedent history of spinal tumour compression. His first presenting symptom of spinal cord compression was an acute onset flaccid paraplegia developing over $24 \mathrm{~h}$. Thomas and Miller ${ }^{4}$ noted that the mean preoperative interval in those with an extradural lipoma was less than 3 years and rarely up to 5 years.

What determines the time at which lipomas with differential growth rates in the same region become symptomatic continues to elude a satisfactory explanation. Why some lipomas accelerate at such a rapid pace and others in the same region progress almost imperceptibly is unclear. It is likely that extradural lipomas may grow over a period of years, leading to slow accommodation without any changes in spinal cord function. However, when the tumor attains a critical volume in the extradural situation, a point may eventually be reached when there is no longer any physiological reserve and static symptoms can progress rapidly, with relative precipitous neurological deterioration. Over the years the slowly growing tumor may cause progressive venous stasis. Examination of the C.S.F in our patient revealed xanthochromia, cytoalbumin dissociation and raised C.S.F pressure which signified spinal venous stasis. The vascular pathology seems to be accelerated when the tumour attains a critical volume in the presence of an existing venous block. This results in catastrophic acute cord ischaemia and rapidly progressive spinal cord compression. No clue to the rapid clinical course manifested in our patient could be found from the histopathological examination of the lipomatous tissue, which was reported to be mature adipose tissue without any evidence of malignancy or dedifferentiation. Thus, we postulate that stasis in the epidural and intradural venous network by a slowly growing lipoma occurred in this patient over a period of time. The mid-thoracic scoliosis could have been the stage of adaptation for early spinal cord compression by the slowly growing lipoma during the asymptomatic disease progression. But, when the lipoma enlarged critically, it caused the rapid onset of complete transverse myelopathy from spinal cord ischaemia.
Associated skeletal, vertebral and spinal abnormalities, C.S.F findings of a spinal block and myelography features have been reported to give no clue towards a pre-operative diagnosis of lipoma. But magnetic resonance (MR) imaging characteristics for spinal cord lipoma are virtually diagnostic. Hence, the specificity of MR imaging can be used for an early and definitive diagnosis prior to severe neurological compromise. Prompt surgical debulking of the tumor can then be done to prevent an unsatisfactory outcome. We emphasize that MRI should be the logical first step for pre-operative diagnosis in symptomatic epidural lipomatosis. ${ }^{9-13}$ Our patient could undergo surgical decompression only after $72 \mathrm{~h}$ of admission (144 h after the ictus) and had possibly suffered irreversible disease progression. Early and timely surgery is the only modality of treatment because neurological function lost before surgery is unlikely to be restored, as was seen in our patient. $^{4,12,13}$

\section{References}

I Mark L et al. Intramedullary spinal cord lipomas. J Neurosurg 1995; 82: $394-400$

2 Knepper PA, McLone DG. Development of spinal cord. In: Park TS, (ed). Spinal dysraphism-Contemporary issues in neurological surgery. 1992; Blackwell Scientific Publications, Boston. pp 1 18.

3 Mclone DG, Naidich TP. Spinal dysraphism Lipomeningocoele and lipoma of the filum terminale. In: Tachdijan MO, (ed). Pediatric Orthopedics, 1990; 3rd edition. W B Saunders, Philadelphia. $1871-1880$.

4 Thomas JE, Miller RH. Lipomatous tumours of the spinal canalA study of their clinical range. Mayo Clin Proc 1973; 48: $393-$ 400 .

5 Giuffre R. Intradural spinal lipoma: review of the literature (99 cases) and report of an additional case. Acta Neurochir 1966; 14: $69-95$.

6 McCormick PC, Stein MB. Intramedullary tumors in the adults. Neurosurg Clin North Am 1990; 1: 609-630.

7 Dyck P. Intramedullary lipoma. Diagnosis and treatment. Spine 1992; 17: $979-981$.

8 Ehni G. Intraspinal and intracranial lipomas (Thesis) Rochester. Minn: Graduate School of Univ of Minnesota, 1943.

9 Fessler RG et al. Epidural lipomatosis in steroid-treated patients. Spine 1992; 17: 183 -- 188.

10 Morano JV, Miller JD, Connors JJ. MR imaging of spinal epidural lipoma. Am J Neuroradiol 1989; 10: 102.

11 Healey ME et al. Demonstration of magnetic resonance of symptomatic spinal epidural lipomatosis. Neurosurgery, 1987; 21: $414-415$.

12 Weidemayer $\mathrm{H}$ et al. Spinal cord compression by extensive epidural lipoma. Eur Neurol 1987; 27: 46-50.

13 Haid RW. Epidural lipomatosis simulating an epidural abscess. Neurosurgery 1987; 21: $744-747$. 